\title{
THE RESTRAINED RAINBOW BONDAGE NUMBER OF A GRAPH
}

\author{
J. AMJADI, N. DEHGARDI, R. KHOEILAR, S. M. SHEIKHOLESLAMI AND L. VOLKMANN
}

\begin{abstract}
A restrained $k$-rainbow dominating function (R $k \mathrm{RDF}$ ) of a graph $G$ is a function $f$ from the vertex set $V(G)$ to the set of all subsets of the set $\{1,2, \ldots, k\}$ such that for any vertex $v \in V(G)$ with $f(v)=\varnothing$ the conditions $\bigcup_{u \in N(v)} f(u)=\{1,2, \ldots, k\}$ and $\mid N(v) \cap$ $\{u \in V \mid f(u)=\varnothing\} \mid \geq 1$ are fulfilled, where $N(v)$ is the open neighborhood of $v$. The weight of a restrained $k$-rainbow dominating function is the value $w(f)=\sum_{\nu \in V}|f(v)|$. The minimum weight of a restrained $k$-rainbow dominating function of $G$ is called the restrained $k$-rainbow domination number of $G$, denoted by $\gamma_{r r k}(G)$. The restrained $k$ rainbow bondage number $b_{r r k}(G)$ of a graph $G$ with maximum degree at least two is the minimum cardinality of all sets $F \subseteq E(G)$ for which $\gamma_{r r k}(G-F)>\gamma_{r r k}(G)$. In this paper, we initiate the study of the restrained $k$-rainbow bondage number in graphs and we present some sharp bounds for $b_{r r 2}(G)$. In addition, we determine the restrained 2rainbow bondage number of some classes of graphs.
\end{abstract}

\section{Introduction}

In this paper, $G$ is a simple graph with vertex set $V(G)$ and edge set $E(G)$ (briefly $V$ and $E)$. For every vertex $v \in V$, the open neighborhood $N(v)$ is the set $\{u \in V(G) \mid u v \in E(G)\}$ and its closed neighborhood is $N[v]=N(v) \cup\{v\}$. Similarly, the open neighborhood of a set $S \subseteq V$ is the set $N(S)=\cup_{v \in S} N(v)$ and its closed neighborhood is $N[S]=N(S) \cup S$. The minimum and maximum degree in $G$ are respectively denoted by $\delta(G)$ and $\Delta(G)$. A vertex of degree one is called a leaf, and its neighbor is called a support vertex. If $v$ is a support vertex, then $L_{v}$ will denote the set of all leaves adjacent to $v$. A support vertex is said to be an end-stem if all its neighbors except one of them are leaves. For $r, s \geq 1$, a double $\operatorname{star} S(r, s)$ is a tree with exactly two vertices that are not leaves, with one adjacent to $r$ leaves and the other to $s$ leaves. For a vertex $v$ in a rooted tree $T$, let $C(v)$ denote the set of children of $v, D(v)$ denote the set of descendants of $v$ and $D[v]=D(v) \cup\{v\}$, and the depth of $v$, $\operatorname{depth}(v)$, is the largest distance from $v$ to a vertex in $D(v)$. The maximal subtree at $v$ is the subtree of $T$ induced by $D(\nu) \cup\{v\}$, and is denoted by $T_{\nu}$. A subset $S$ of vertices of $G$ is a dominating set if $N[S]=V$.

Received December 2, 2016, accepted December 27, 2017.

2010 Mathematics Subject Classification. 05C69.

Key words and phrases. $k$-rainbow domination number, restrained $k$-rainbow domination number, restrained $k$-rainbow bondage number.

Corresponding author: J. Amjadi. 
The domination number $\gamma(G)$ is the minimum cardinality of a dominating set of $G$. For a more thorough treatment of domination parameters and for terminology not presented here see $[17,22]$.

A subset $S$ of vertices of $G$ is a restrained dominating set if $N[S]=V$ and the subgraph induced by $V-S$ has no isolated vertex. The restrained domination number $\gamma_{r}(G)$ is the minimum cardinality of a restrained dominating set of $G$. The restrained domination number was introduced by Domke et al. [14] and has been studied by several author (see for example $[12,13])$. The restrained bondage number $b_{r}(G)$ of a nonempty graph $G$ is the minimum cardinality among all sets of edges $F \subseteq E(G)$ for which $\gamma_{r}(G-F)>\gamma_{r}(G)$. The restrained bondage number has been investigated in $[15,18]$.

For a positive integer $k$, a $k$-rainbow dominating function (kRDF) of a graph $G$ is a function $f$ from the vertex set $V(G)$ to the set of all subsets of the set $\{1,2, \ldots, k\}$ such that for any vertex $v \in V(G)$ with $f(v)=\varnothing$ the condition $\bigcup_{u \in N(v)} f(u)=\{1,2, \ldots, k\}$ is fulfilled. The weight of a $\operatorname{kRDF} f$ is the value $w(f)=\sum_{v \in V}|f(v)|$. The $k$-rainbow domination number of a graph $G$, denoted by $\gamma_{r k}(G)$, is the minimum weight of a $\mathrm{kRDF}$ of $G$. Note that $\gamma_{r 1}(G)$ is the classical domination number $\gamma(G)$. The $k$-rainbow domination number was introduced by Brešar, Henning, and Rall [7] and has been studied by several authors (see for example $[2,3,8,9,10,19,20,21,23,24])$.

A $k$-rainbow dominating function $f$ is called a restrained $k$-rainbow dominating function (RkRDF) if the induced subgraph $G[\{v \in V \mid f(v)=\varnothing\}]$ has no isolated vertex. The restrained $k$-rainbow domination number of $G$, denoted by $\gamma_{r r k}(G)$, is the minimum weight of an $\mathrm{R} k \mathrm{RDF}$ on $G$. A $\gamma_{r r k}(G)$-function is an $\operatorname{RkRDF}$ of $G$ with $\omega(f)=\gamma_{r r k}(G)$. If $f$ is a $\gamma_{r r k}(G)$-function, then since $V-\{v \in V \mid f(v)=\varnothing\}$ is a restrained dominating set, and since placing $\{1,2, \ldots, k\}$ at the vertices of a restrained dominating set yields an $\mathrm{R} k \mathrm{RDF}$, we have

$$
\max \left\{\gamma_{r k}(G), \gamma_{r}(G)\right\} \leq \gamma_{r r k}(G) \leq k \gamma_{r}(G)
$$

The restrained $k$-rainbow domination number has been investigated in $[1,5]$.

Let $G$ be a graph of order $n \geq k+1$ with $\gamma_{r r k}(G)<n$. The restrained $k$-rainbow bondage number $b_{r r k}(G)$ of $G$ is the minimum cardinality of all sets $E^{\prime} \subseteq E$ for which $\gamma_{r r k}\left(G-E^{\prime}\right)>$ $\gamma_{r r k}(G)$. An edge set $B$ with $\gamma_{r r k}(G-B)>\gamma_{r r k}(G)$ is called the restrained $k$-rainbow bondage set. A $b_{r r k}(G)$-set is a restrained $k$-rainbow bondage set of $G$ of size $b_{r r k}(G)$. If $B$ is a $b_{r r k}(G)$ set, then clearly $\gamma_{r r k}(G-B)=\gamma_{r r k}(G)+1$.

The $k$-rainbow bondage number $b_{r k}(G)$ for usual $k$-rainbow domination number was introduced by Dehgardi et al. in [11] and has been studied by several authors [4, 6].

One possible application of the concept of $k$-rainbow restrained domination is that of cities and emergency guards. Here, every vertex with a positive weight in a $k$-rainbow restrained dominating function, corresponds to a position of an emergency guard and each 
vertex not occupied by an emergency guard corresponds to a position of a city without any emergency guards, which is adjacent to at least one other deprived city. The $k$-rainbow restrained bondage number measures the vulnerability of the connection between situations under unpredictable events or attacks. The minimum $k$-rainbow restrained dominating function of cities plays an important role for dominating the whole situations with the minimum cost. So, we must consider whether its function remains safe under the unpredictable event or attack. Suppose that an unpredictable event happens. Then how many connection routes does it have to destroy so that the cost can not remains the same in order to $k$-rainbow restrained dominate the whole city? The minimum number of connection routes is just the $k$-rainbow restrained bondage number.

Our purpose in this paper is to initiate the study of the restrained $k$-rainbow bondage number in graphs. We first establish some sharp bounds for the restrained $k$-rainbow bondage number of a graph. In particular, we prove that for any tree $T$ of order $n \geq 5$ with $\operatorname{diam}(T) \geq 3$ and different from $P_{5}, P_{6}, b_{r r 2}(T) \leq(n-3) / 2$. In addition, we determine the restrained 2rainbow bondage number of some classes of graphs.

We make use of the following results in this paper.

Theorem A ([5]). For $n \geq 4, \gamma_{r r 2}\left(P_{n}\right)=\left\lceil\frac{2 n+1}{3}\right\rceil+1$ and $\gamma_{r r 2}\left(P_{n}\right)=n$ otherwise.

Corollary 1.1. For $n \geq 7, b_{r r 2}\left(P_{n}\right)=1$.

Proof. Let $P_{n}:=v_{1} v_{2} \ldots v_{n}$. It follows from Theorem A that

$$
\gamma_{r r 2}\left(P_{n}-v_{3} v_{4}\right)=\gamma_{r r 2}\left(P_{n-3}\right)+3=\left\lceil\frac{2(n-3)+1}{3}\right\rceil+1+3>\left\lceil\frac{2 n+1}{3}\right\rceil+1=\gamma_{r r 2}\left(P_{n}\right) .
$$

Hence $b_{r r 2}\left(P_{n}\right)=1$.

Theorem B ([5]). For $n \geq 6, \gamma_{r r 2}\left(C_{n}\right)=2\left\lceil\frac{n}{3}\right\rceil+1$ when $n \equiv 2(\bmod 3)$ and $\gamma_{r r 2}\left(C_{n}\right)=2\left\lceil\frac{n}{3}\right\rceil$ otherwise.

Corollary 1.2. For $n \geq 6$,

$$
b_{r r 2}\left(C_{n}\right)= \begin{cases}1 & \text { if } n \equiv 0(\bmod 3) \\ 2 & \text { otherwise }\end{cases}
$$

Proof. Let $C_{n}:=\left(v_{1} v_{2} \ldots v_{n}\right)$. If $n=3 k(k \geq 2)$, then it follows from Theorem A and B that

$$
\gamma_{r r 2}\left(C_{n}-v_{1} v_{n}\right)=\gamma_{r r 2}\left(P_{n}\right)=\left\lceil\frac{2 n+1}{3}\right\rceil+1>2\left\lceil\frac{n}{3}\right\rceil=\gamma_{r r 2}\left(C_{n}\right) .
$$


Hence $b_{r r 2}\left(C_{n}\right)=1$ if $n \equiv 0(\bmod 3)$. Now let $n \equiv 1(\bmod 3)$. Then obviously $\left\lceil\frac{2 n+1}{3}\right\rceil+1=$ $2\left\lceil\frac{n}{3}\right\rceil$ that implies $b_{r r 2}\left(C_{n}\right) \geq 2$ in this case. On the other hand, Theorems A and B imply that

$$
\gamma_{r r 2}\left(C_{n}-\left\{v_{1} v_{2}, v_{4} v_{5}\right\}\right)=\gamma_{r r 2}\left(P_{n-3}\right)+3=\left\lceil\frac{2(n-3)+1}{3}\right\rceil+1+3>2\left\lceil\frac{n}{3}\right\rceil=\gamma_{r r 2}\left(C_{n}\right) .
$$

Hence $b_{r r 2}\left(C_{n}\right)=2$ if $n \equiv 1(\bmod 3)$. Finally let $n \equiv 2(\bmod 3)$. It is easy to see that $\left\lceil\frac{2 n+1}{3}\right\rceil+$ $1=2\left\lceil\frac{n}{3}\right\rceil+1$ which implies that $b_{r r 2}\left(C_{n}\right) \geq 2$ in this case. We deduce from Theorems A and B that

$$
\gamma_{r r 2}\left(C_{n}-\left\{v_{1} v_{2}, v_{4} v_{5}\right\}\right)=\gamma_{r r 2}\left(P_{n-3}\right)+3=\left\lceil\frac{2(n-3)+1}{3}\right\rceil+1+3>2\left\lceil\frac{n}{3}\right\rceil+1=\gamma_{r r 2}\left(C_{n}\right)
$$

and so $b_{r r 2}\left(C_{n}\right)=2$ in this case. This completes the proof.

Theorem C ([5]). Let $G$ be a connected graph of order $n \geq 2$. Then $\gamma_{r r 2}(G)=n$ if and only if $G \simeq K_{1, n-1}, C_{4}, C_{5}$ or $G=P_{n}$ for $n=2,3,4,5,6$.

Theorem $\mathbf{D}([5])$. Let $G$ be a graph of order $n \geq 2$. Then $\gamma_{r r 2}(G)=2$ if and only if $n=2$ or $n \geq 3$ and $2 \leq \delta(G) \leq \Delta(G)=n-1$ or $3 \leq \delta(G) \leq \Delta(G)=n-2$ and there exist two distinct vertices $u$ and $v$ such that $V(G)-\{u, v\} \subseteq N(u) \cap N(v)$.

Theorem $\mathbf{E}([11])$. If $k \geq 2$ is an integer and $n \geq k+1$, then $b_{r k}\left(K_{n}\right)=\left\lceil\frac{k n}{k+1}\right\rceil$.

Theorem $\mathbf{F}([11])$. Let $G$ be a graph of order $n \geq 3$. Then $\gamma_{r 2}(G)=2$ if and only if there exists a vertex set $A$ with $|A| \leq 2$ such that every vertex of $V(G)-A$ is adjacent to every vertex of $A$.

\section{Bounds on the restrained rainbow bondage number}

In this section we first establish a sharp upper bound on the restrained 2-rainbow bondage number of trees in terms of their order and then we present two sharp bounds on the restrained 2-rainbow bondage number of general graphs.

Observation 2.1. If $T=S(r, s)$ is a double star of order $r+s+2 \geq 5$, then $b_{r r 2}(T)=1$.

Theorem 2.2. Let $T$ be a tree of order $n \geq 5$. If $\operatorname{diam}(T) \geq 3$ and $T \notin\left\{P_{5}, P_{6}\right\}$, then

$$
b_{r r 2}(T) \leq \frac{n-3}{2}
$$

Furthermore, this bound is sharp. 
Proof. If $\operatorname{diam}(T)=3$, then $T$ is a double star of order at least 5 and it follows from Observation 2.1 that $b_{r r 2}(T)=1 \leq \frac{n-3}{2}$. Assume that $\operatorname{diam}(T) \geq 4$. Let $P=v_{1} v_{2} \ldots v_{d}$ be a diametral path in $T$ such that $\operatorname{deg}\left(v_{2}\right)$ is as large as possible. Among all paths with this property we choose a path such that $\left|L_{v_{3}}\right|$ is as large as possible. Root $T$ at $v_{d}$. We consider the following cases.

Case 1. $\operatorname{deg}\left(v_{2}\right) \geq 3$.

Suppose that $u \in L_{v_{2}}-\left\{v_{1}\right\}$. First let $\operatorname{deg}\left(v_{3}\right) \geq 3$. Assume that $L_{v_{3}}=\left\{x_{1}, x_{2}, \ldots, x_{k}\right\}$ if $L_{\nu_{3}} \neq \varnothing$ and $N\left(v_{3}\right) \backslash\left(L_{v_{3}} \cup\left\{v_{2}, v_{4}\right\}\right)=\left\{y_{1}, y_{2}, \ldots, y_{t}\right\}$ when $N\left(v_{3}\right) \backslash\left(L_{v_{3}} \cup\left\{v_{2}, v_{4}\right\}\right) \neq \varnothing$. We consider two subcases.

Subcase 1.1. $L_{v_{3}} \neq \varnothing$.

Let $B=\left\{v_{3} y_{1}, \ldots, v_{3} y_{t}, v_{3} v_{2}, v_{3} v_{4}\right\}$. Clearly, $n \geq 2 t+7$ and so

$$
|B|=t+2 \leq(n-7) / 2+2 \leq(n-3) / 2 .
$$

Let $T_{1}, T_{2}, \ldots, T_{t}, T_{t+1}, T_{t+2}$ be the components of $T-B$ containing $y_{1}, y_{2}, \ldots, y_{t}, v_{2}, v_{3}$ respectively. Assume that $f$ is a $\gamma_{r r 2}(T-B)$-function. Then clearly $|f(x)|=1$ for every vertex $x \in$ $\bigcup_{i=1}^{t+2} V\left(T_{i}\right)$. Define $g: V(T) \rightarrow \mathscr{P}(\{1,2\})$ by $g\left(v_{1}\right)=\{1\}, g(u)=\{2\}, g\left(x_{1}\right)=\{1,2\}, g\left(v_{2}\right)=g\left(v_{3}\right)=$ $\varnothing$, and $g(x)=f(x)$ otherwise. It is easy to see that $g$ is a restrained 2-rainbow dominating function of $T$ of weight less than $\omega(f)$ and hence $b_{r r 2}(T) \leq|B| \leq(n-3) / 2$ as desired.

Subcase 1.2. $L_{v_{3}}=\varnothing$.

Since $\operatorname{deg}\left(v_{3}\right) \geq 3$, we have $t \geq 1$. Assume that $B=\left\{v_{3} y_{1}, \ldots, v_{3} y_{t}, v_{3} v_{4}\right\}$. Then clearly $n \geq 2 t+6$ and so

$$
|B|=t+1 \leq(n-6) / 2+1<(n-3) / 2 .
$$

Let $T_{1}, T_{2}, \ldots, T_{t}, T_{t+1}$ be the components of $T-B$ containing $y_{1}, y_{2}, \ldots, y_{t}, v_{3}$ respectively, and $f$ be a $\gamma_{r r 2}(T-B)$-function. Then $|f(x)|=1$ for every vertex $x \in \bigcup_{i=1}^{t+1} V\left(T_{i}\right)$. Define $g: V(T) \rightarrow$ $\mathscr{P}(\{1,2\})$ by $g\left(v_{1}\right)=\{1\}, g(u)=\{2\}, g\left(y_{1}\right)=\{1,2\}, g\left(v_{2}\right)=g\left(v_{3}\right)=\varnothing$ and $g(x)=f(x)$ otherwise. Obviously, $g$ is a restrained 2-rainbow dominating function of $T$ of weight less than $\omega(f)$ and hence $b_{r r 2}(T) \leq|B|<(n-3) / 2$.

Now let $\operatorname{deg}\left(\nu_{3}\right)=2$. By the choice of the diametral path, every vertex with depth 2 in $N\left(v_{4}\right)-\left\{v_{5}\right\}$, have degree 2 . If $n=6$ or 7 , then clearly $b_{r r 2}(T)=1<(n-3) / 2$ and we are done. Suppose that $n \geq 8$. If $\operatorname{deg}\left(v_{4}\right)=2$ or $N\left(v_{4}\right) \backslash\left\{v_{3}, v_{5}\right\}=L_{v_{4}}$, then let $B=\left\{v_{5} v_{4}, v_{4} v_{3}\right\}, T_{1}, T_{2}$ be the components of $T-B$ containing $v_{4}, v_{3}$, respectively, and $f$ be a $\gamma_{r r 2}(T-B)$-function. Clearly, $|f(x)|=1$ for each $x \in V\left(T_{1}\right) \cup V\left(T_{2}\right)$. Then the function $g: V(T) \rightarrow \mathscr{P}(\{1,2\})$ defined by $g\left(v_{4}\right)=$ $\{1,2\}, g(u)=\{1\}, g\left(v_{1}\right)=\{2\}, g\left(v_{2}\right)=g\left(v_{3}\right)=\varnothing$ and $g(x)=f(x)$ otherwise, is a restrained 2rainbow dominating function of $T$ of weight less than $\omega(f)$ and hence $b_{r r 2}(T) \leq 2<(n-3) / 2$ as desired. Henceforth, we assume that $\operatorname{deg}\left(v_{4}\right) \geq 3$ and $v_{4}$ has a neighbor of degree at least two other than $v_{3}, v_{5}$. Let $N\left(v_{4}\right)-\left\{v_{3}, v_{5}\right\}=L_{v_{4}} \cup\left\{y_{1}, y_{2}, \ldots, y_{t}\right\}$. Clearly, $n \geq 2 t+6$. If $n \geq 2 t+7$ 
then let $B=\left\{v_{4} y_{1}, \ldots, v_{4} y_{t}, v_{3} v_{4}, v_{4} v_{5}\right\}$, and if $n=2 t+6$ then let $B=\left\{v_{4} y_{2}, \ldots, v_{4} y_{t}, v_{3} v_{4}, v_{4} v_{5}\right\}$. Then $|B| \leq(n-3) / 2$. Assume that $f$ is a $\gamma_{r r 2}(T-B)$-function. Clearly, $|f(x)|=1$ for each $x \in$ $V\left(T_{v_{4}}\right)$. Define $g: V(T) \rightarrow \mathscr{P}(\{1,2\})$ by $g\left(v_{4}\right)=\{1,2\}, g\left(v_{1}\right)=\{1\}, g(u)=\{2\}, g\left(v_{2}\right)=g\left(v_{3}\right)=\varnothing$, and $g(x)=f(x)$ otherwise. Obviously, $g$ is a restrained 2-rainbow dominating function of $T$ of weight less than $\omega(f)$ and hence $b_{r r 2}(T) \leq|B| \leq(n-3) / 2$.

Case 2. $\operatorname{deg}\left(v_{2}\right)=2$.

By the choice of the diametral path, we deduce that every end-steam on a diametral path has degree 2. In particular, any child of $v_{3}$ is a leaf or a support vertex of degree 2 . Consider the following subcases.

Subcase 2.1. $\operatorname{deg}\left(v_{3}\right) \geq 3$.

Assume that $L_{\nu_{3}}=\left\{x_{1}, x_{2}, \ldots, x_{k}\right\}$ if $L_{v_{3}} \neq \varnothing$ and $N\left(v_{3}\right)-\left\{v_{4}, v_{2}\right\}=L_{v_{3}} \cup\left\{y_{1}, y_{2}, \ldots, y_{t}\right\}$ when $N\left(v_{3}\right)-\left\{v_{4}, v_{2}\right\} \neq L_{v_{3}}$. We distinguish the following.

- $N\left(v_{3}\right)-\left\{v_{4}, v_{2}\right\}=L_{v_{3}}$.

If $n=6$, then clearly $b_{r r 2}(T)=1<\frac{n-3}{2}$. Hence, we assume that $n \geq 7$. Let $T^{\prime}=T-$ $\left\{v_{4} v_{3}, v_{3} v_{2}\right\}$ and $f$ be a $\gamma_{r r 2}\left(T^{\prime}\right)$-function. Clearly, $|f(x)|=1$ for each vertex $x \in V\left(T_{\nu_{3}}\right)$. If $f\left(v_{4}\right)=\varnothing$, then define the function $g: V(T) \rightarrow \mathscr{P}(\{1,2\})$ by $g\left(x_{1}\right)=\{1\}, g\left(\nu_{3}\right)=\varnothing, g\left(v_{2}\right)=$ $\{2\}$ and $g(x)=f(x)$ otherwise. If $f\left(\nu_{4}\right) \neq \varnothing$, then let, without loss of generality, $1 \in f\left(v_{4}\right)$ and define the function $g: V(T) \rightarrow \mathscr{P}(\{1,2\})$ by $g\left(x_{1}\right)=\{2\}, g\left(v_{1}\right)=\{1,2\}, g\left(v_{2}\right)=g\left(v_{3}\right)=$ $\varnothing$ and $g(x)=f(x)$ otherwise. It is easy to see that $g$ is a restrained 2-rainbow dominating function of $T$ of weight less than $\omega(f)$ and hence $b_{r r 2}(T) \leq 2 \leq(n-3) / 2$.

- $N\left(v_{3}\right)-\left\{v_{4}, v_{2}\right\} \neq L_{v_{3}}$.

First let $\left|L_{v_{3}}\right| \geq 2$. Assume that $B=\left\{v_{3} y_{1}, \ldots, v_{3} y_{t}, v_{3} \nu_{2}, v_{3} v_{4}\right\}, f$ is a $\gamma_{r r 2}(T-B)$-function and $T_{1}, T_{2}, \ldots, T_{t}, T_{t+1}, T_{t+2}$ are the components of $T-B$ containing $y_{1}, \ldots, y_{t}, v_{2}, v_{3}$ respectively. Clearly, $|B| \leq(n-3) / 2$ and $|f(x)|=1$ for each $x \in \bigcup_{i=1}^{t+2} V\left(T_{i}\right)$. Define the function $g: V(T) \rightarrow \mathscr{P}(\{1,2\})$ by $g\left(x_{1}\right)=\{1\}, g\left(x_{2}\right)=\{2\}, g\left(\nu_{1}\right)=\{1,2\}, g\left(\nu_{2}\right)=g\left(\nu_{3}\right)=\varnothing$ and $g(x)=f(x)$ otherwise. Obviously, $g$ is a restrained 2-rainbow dominating function of $T$ of weight less than $\omega(f)$ and hence $b_{r r 2}(T) \leq|B| \leq(n-3) / 2$.

Now let $\left|L_{v_{3}}\right| \leq 1$. Assume that $B=\left\{v_{3} y_{1}, \ldots, v_{3} y_{t}, v_{3} v_{4}\right\}$ and $f$ is a $\gamma_{r r 2}(T-B)$-function. Obviously, $|B| \leq(n-3) / 2$ and $|f(x)|=1$ for each $x \in V\left(T_{\nu_{3}}\right)$. If $f\left(v_{4}\right)=\varnothing$, then the function $g: V(T) \rightarrow \mathscr{P}(\{1,2\})$ defined by $g\left(y_{1}\right)=\{1\}, g\left(\nu_{2}\right)=\{2\}, g\left(\nu_{3}\right)=\varnothing$ and $g(x)=f(x)$ otherwise, is a restrained 2-rainbow dominating function of $T$ of weight less than $\omega(f)$, implying that $b_{r r 2}(T) \leq|B| \leq(n-3) / 2$. If $f\left(v_{4}\right) \neq \varnothing$, then let, without loss of generality, $1 \in f\left(v_{4}\right)$ and define $g: V(T) \rightarrow \mathscr{P}(\{1,2\})$ by $g\left(y_{1}\right)=\{2\}, g\left(\nu_{1}\right)=\{1,2\}, g\left(\nu_{2}\right)=g\left(\nu_{3}\right)=\varnothing$ and $g(x)=f(x)$ otherwise. Clearly, $g$ is a restrained 2-rainbow dominating function of $T$ of weight less than $\omega(f)$ and hence $b_{r r 2}(T) \leq(n-3) / 2$. 
Subcase 2.2. $\operatorname{deg}\left(v_{3}\right)=2$.

By the choice of the diametral path, we deduce that every vertex with depth 2 on a diametral path has degree 2. Since $T \notin\left\{P_{5}, P_{6}\right\}$, we must have $\operatorname{diam}(T) \geq 6$. Let $L_{v_{4}}=\left\{x_{1}, x_{2}, \ldots, x_{k}\right\}$ if $L_{v_{4}} \neq \varnothing$ and $N\left(v_{4}\right)-\left\{v_{5}, v_{3}\right\}=L_{v_{4}} \cup\left\{y_{1}, y_{2}, \ldots, y_{t}\right\}$ when $N\left(v_{4}\right)-\left\{v_{5}, v_{3}\right\} \neq L_{v_{4}}$. We distinguish the following.

(a) $\operatorname{deg}\left(v_{4}\right) \geq 3$ and $N\left(v_{4}\right)-\left\{v_{5}, v_{3}\right\}=L\left(v_{4}\right)$.

Then $k \geq 1$. If $k \geq 2$, then it is easy to see that $\gamma_{r r 2}\left(T-\left\{v_{5} v_{4}, v_{4} v_{3}\right\}\right)>\gamma_{r r 2}(T)$ that implies $b_{r r 2}(T) \leq 2 \leq(n-3) / 2$. Let $k=1, T^{\prime}=T-v_{5} \nu_{4}$ and let $f$ be a $\gamma_{r r 2}\left(T^{\prime}\right)$-function. If $f\left(\nu_{5}\right)=$ $\varnothing$, then the function $g: V(T) \rightarrow \mathscr{P}(\{1,2\})$ defined by $g\left(v_{3}\right)=\{1\}, g\left(x_{1}\right)=\{2\}, g\left(v_{4}\right)=\varnothing$ and $g(x)=f(x)$ otherwise, is a restrained 2-rainbow dominating function of $T$ of weight less than $\omega(f)$ and hence $b_{r r 2}(T)=1<\frac{n-3}{2}$. If $f\left(\nu_{5}\right) \neq \varnothing$, then let, without loss of generality, $1 \in f\left(\nu_{5}\right)$ and define $g: V(T) \rightarrow \mathscr{P}(\{1,2\})$ by $g\left(\nu_{2}\right)=\{1,2\}, g\left(x_{1}\right)=\{2\}, g\left(\nu_{3}\right)=g\left(\nu_{4}\right)=\varnothing$ and $g(x)=f(x)$ otherwise. Clearly, $g$ is a restrained 2-rainbow dominating function of $T$ of weight less than $\omega(f)$ and so $b_{r r 2}(T)=1<(n-3) / 2$.

(b) $\operatorname{deg}\left(v_{4}\right) \geq 3, N\left(v_{4}\right)-\left\{v_{5}, v_{3}\right\} \neq L_{v_{4}}$ and $\operatorname{deg}\left(y_{j}\right) \geq 3$ for some $j$.

Let $j=1, B=\left\{v_{4} y_{1}, \ldots, v_{4} y_{t}, v_{3} v_{4}, v_{5} v_{4}\right\}$ and $f$ be a $\gamma_{r r 2}(T-B)$-function. Clearly, $|B|<$ $(n-3) / 2$ and $|f(x)|=1$ for each $x \in V\left(T_{\nu_{4}}\right)$. Let $w_{1}, w_{2} \in N\left(y_{1}\right)-\left\{v_{4}\right\}$ and define $g: V(T) \rightarrow$ $\mathscr{P}(\{1,2\})$ by $g\left(w_{1}\right)=\{1\}, g\left(w_{2}\right)=\{2\}, g\left(\nu_{3}\right)=\{1,2\}, g\left(v_{4}\right)=g\left(y_{1}\right)=\varnothing$ and $g(x)=f(x)$ otherwise. It is easy to see that $g$ is a restrained 2-rainbow dominating function of $T$ of weight less than $\omega(f)$ and hence $b_{r r 2}(T)<(n-3) / 2$.

(c) $\operatorname{deg}\left(v_{4}\right) \geq 3, N\left(v_{4}\right)-\left\{v_{5}, v_{3}\right\} \neq L_{v_{4}}$ and $\operatorname{deg}\left(y_{j}\right)=2$ for each $j$.

Let $B=\left\{v_{4} y_{1}, \ldots, v_{4} y_{t}, v_{3} v_{4}, v_{5} v_{4}\right\}, f$ a $\gamma_{r r 2}(T-B)$-function and $T_{1}, T_{2}, \ldots, T_{t}, T_{t+1}, T_{t+2}$ be the components of $T-B$ containing $y_{1}, \ldots, y_{t}, v_{3}, v_{4}$, respectively. Then $|f(x)|=1$ for each $x \in \bigcup_{i=1}^{t+2} V\left(T_{i}\right)$ and $|B| \leq(n-3) / 2$ because $n \geq 2 t+7$. If $f\left(\nu_{5}\right)=\varnothing$, then the function $g$ : $V(T) \rightarrow \mathscr{P}(\{1,2\})$ defined by $g\left(\nu_{3}\right)=\{1\}, g\left(y_{1}\right)=\{2\}, g\left(v_{4}\right)=\varnothing$ and $g(x)=f(x)$ otherwise, is a restrained 2-rainbow dominating function of $T$ of weight less than $\omega(f)$ and hence $b_{r r 2}(T) \leq(n-3) / 2$. If $f\left(\nu_{5}\right) \neq \varnothing$, then let, without loss of generality, $1 \in f\left(\nu_{5}\right)$ and define $g$ : $V(T) \rightarrow \mathscr{P}(\{1,2\})$ by $g\left(\nu_{2}\right)=\{1,2\}, g\left(y_{1}\right)=\{2\}, g\left(\nu_{3}\right)=g\left(v_{4}\right)=\varnothing$ and $g(x)=f(x)$ otherwise. Clearly, $g$ is a restrained 2-rainbow dominating function of $T$ of weight less than $\omega(f)$ that implies $b_{r r 2}(T) \leq(n-3) / 2$.

(d) $\operatorname{deg}\left(v_{4}\right)=2$.

Considering above, we may assume that any maximal subtree at each child of $v_{5}$ with depth 3 , is the path $P_{4}$. Consider the following.

(i) $\operatorname{deg}\left(v_{5}\right) \geq 3$.

Assume that $N\left(v_{5}\right)-\left\{v_{6}, v_{4}\right\}=L_{v_{5}} \cup\left\{y_{1}, y_{2}, \ldots, y_{t}\right\}$ if $N\left(v_{5}\right)-\left\{v_{6}, v_{4}\right\} \neq L_{v_{5}}$ and $L_{v_{5}}=$ $\left\{x_{1}, x_{2}, \ldots, x_{k}\right\}$ when $L_{v_{5}} \neq \varnothing$. 
- $L_{v_{5}} \neq \varnothing$.

If $N\left(\nu_{5}\right)-\left\{v_{6}, v_{4}\right\}=L_{\nu_{5}}$, then it is easy to see that $\gamma_{r r 2}\left(T-\left\{v_{5} v_{6}, v_{5} \nu_{4}\right\}\right)>\gamma_{r r 2}(T)$ that implies $b_{r r 2}(T) \leq 2<(n-3) / 2$. Assume that $N\left(\nu_{5}\right)-\left\{v_{6}, v_{4}\right\} \neq L_{\nu_{5}}$ and $B=\left\{v_{5} y_{1}, \ldots, v_{5} y_{t}, v_{5} v_{4}, v_{5} v_{6}\right\}$. As above $|B|<(n-3) / 2$. Let $f$ be a $\gamma_{r r 2}(T-B)-$ function. Then we may assume that $\left|f\left(\nu_{i}\right)\right|=\left|f\left(x_{j}\right)\right|=1$ for $1 \leq i \leq 5$ and $1 \leq$ $j \leq k$. Using an argument similar to that described in Case 3 , we can see that $\gamma_{r r 2}(T)<\gamma_{r r 2}(T-B)$ implying that $b_{r r 2} \leq(n-3) / 2$.

- $L_{v_{5}}=\varnothing$ and $T_{y_{i}}$ has a $\gamma_{r r 2}\left(T_{y_{i}}\right)$-function $h$ such that $\left|h\left(y_{i}\right)\right| \geq 1$ for some $i$. Assume, without loss of generality, that $j=1$ and $1 \in h\left(y_{1}\right)$. Let $B=\left\{v_{5} y_{1}, \ldots, v_{5} y_{t}\right.$, $\left.\nu_{5} v_{6}\right\}, f$ be a $\gamma_{r r 2}(T-B)$-function and $T_{1}, T_{2}, \ldots, T_{t}, T_{t+1}, T_{t+2}$ be the components of $T-B$ containing $y_{1}, \ldots, y_{t}, v_{6}, v_{5}$ respectively. We may assume that $\left.f\right|_{y_{1}}$ $=h$ and $\left|f\left(v_{i}\right)\right|=1$ for each $1 \leq i \leq 5$. If there exists a vertex $w \in\left\{v_{6}, y_{2}, \ldots, y_{t}\right\}$ with $f(w)=\varnothing$, then define $g: V(T) \rightarrow \mathscr{P}(\{1,2\})$ by $g\left(v_{4}\right)=\{2\}, g\left(\nu_{5}\right)=\varnothing$ and $g(x)=f(x)$ otherwise. Clearly, $g$ is a restrained 2-rainbow dominating function of $T$ of weight less than $\omega(f)$ and so $b_{r r 2}(T) \leq(n-3) / 2$. Assume that $|f(w)| \geq 1$ for each $w \in\left\{v_{6}, y_{1}, \ldots, y_{t}\right\}$. Suppose, without loss of generality, that $2 \in f\left(v_{6}\right)$. Then the function $g: V(T) \rightarrow \mathscr{P}(\{1,2\})$ defined by $g\left(v_{5}\right)=g\left(v_{4}\right)=\varnothing, g\left(\nu_{3}\right)=\{1,2\}$ and $g(x)=f(x)$ otherwise, is a restrained 2-rainbow dominating function of $T$ of weight less than $\omega(f)$ and so $b_{r r 2}(T) \leq|B| \leq(n-3) / 2$.

- $L_{\nu_{5}}=\varnothing$ and For each $i$ and each $\gamma_{r r 2}\left(T_{y_{i}}\right)$-function $h, h\left(y_{i}\right)=\varnothing$.

Then $\operatorname{deg}_{T_{y_{1}}}\left(y_{1}\right) \geq 2$ and $y_{1}$ has a neighbor of degree at least two with exception of $v_{5}$. Let $N\left(y_{1}\right) \backslash\left(L_{y_{1}} \cup\left\{v_{5}\right\}\right)=\left\{z_{1}, z_{2}, \ldots, z_{s}\right\}$. If the component of $T-v_{6} v_{5}$ containing $v_{6}$, has a $\gamma_{r r 2}$-function $f^{\prime}$ with $\left|f^{\prime}\left(v_{6}\right)\right| \geq 1$, then as above we can see that $b_{r r 2}(T) \leq(n-3) / 2$. Hence, we assume that every $\gamma_{r r 2}$-function of the component of $T-v_{6} v_{5}$ containing $v_{6}$, assigns $\varnothing$ to $v_{6}$. Now let $B=\left\{v_{5} y_{1}, \ldots, v_{5} y_{t}, v_{5} v_{6}\right.$, $\left.y_{1} z_{1}, \ldots, y_{1} z_{s}\right\}, f$ be a $\gamma_{r r 2}(T-B)$-function and $T_{1}$ be the component of $T-B$ containing $y_{1}$. Obviously, $n \geq 2 t+2 s+6$ and so $|B|<(n-3) / 2$. On the other hand, we may assume that $\left|f\left(y_{1}\right)\right|=1$ and $\left|f\left(v_{i}\right)\right|=1$ for $1 \leq i \leq 5$. Let $h$ be a $\gamma_{r r 2}\left(T_{y_{1}}\right)$-function. By assumption we have $h\left(y_{1}\right)=\varnothing$ and $\sum_{x \in V\left(T_{y_{1}}\right)}|f(x)|>$ $\sum_{x \in V\left(T_{y_{1}}\right)}|h(x)|$. Then the function $g: V(T) \rightarrow \mathscr{P}(\{1,2\})$ defined by $g\left(\nu_{5}\right)=g\left(\nu_{3}\right)$ $=g\left(v_{2}\right)=\varnothing, g\left(v_{4}\right)=g\left(v_{1}\right)=\{1,2\}, g(u)=h(u)$ for $u \in V\left(T_{y_{1}}\right)$ and $g(u)=f(u)$ otherwise, is a restrained 2-rainbow dominating function of $T$ of weight less than $\omega(f)$ and so $b_{r r 2}(T) \leq|B| \leq \frac{n-3}{2}$.

(ii) $\operatorname{deg}\left(\nu_{5}\right)=2$.

Considering the above cases and subcases, we have $\operatorname{deg}\left(v_{i}\right)=2$ for $2 \leq i \leq 5$. Similarly, we may assume that $\operatorname{deg}\left(v_{i}\right)=2$ for $d-4 \leq i \leq d-1$. Using an argument similar to that described in the proof of (i), we can show that $b_{r r 2}(T) \leq \frac{n-3}{2}$ when 
$\operatorname{deg}\left(v_{6}\right) \geq 3$. Therefore, we suppose that $\operatorname{deg}\left(v_{6}\right)=2$. If $\operatorname{deg}\left(v_{7}\right)=2$, then it is easy to see that $\gamma_{r r 2}(T)<\gamma_{r r 2}\left(T-\left\{v_{7} v_{8}, v_{7} v_{6}\right\}\right)$ that implies $b_{r r 2}(T) \leq 2 \leq(n-3) / 2$. Hence, we assume $\operatorname{deg}\left(\nu_{7}\right) \geq 3$. Let $N\left(\nu_{7}\right)-\left\{v_{8}, v_{6}\right\}=L_{\nu_{7}} \cup\left\{y_{1}, \ldots, y_{t}\right\}$ if $N\left(\nu_{7}\right)-\left\{v_{8}, v_{6}\right\} \neq L_{v_{7}}$ and $L_{v_{7}}=\left\{x_{1}, \ldots, x_{k}\right\}$ when $L_{v_{7}} \neq \varnothing$. Suppose that $B=\left\{v_{7} y_{1}, \ldots, v_{7} y_{t}, v_{7} v_{6}, v_{7} v_{8}\right\}$ and $f$ is a $\gamma_{r r 2}(T-B)$-function. Clearly, $|B|<(n-3) / 2$ and we may assume that $\left|f\left(v_{i}\right)\right|=1$ for $1 \leq i \leq 7$. Then the function $g: V(T) \rightarrow \mathscr{P}(\{1,2\})$ defined by $g\left(v_{7}\right)=$ $g\left(\nu_{4}\right)=g\left(\nu_{1}\right)=\{1,2\}, g\left(v_{6}\right)=g\left(\nu_{5}\right)=g\left(\nu_{3}\right)=g\left(\nu_{2}\right)=\varnothing$ and $g(x)=f(x)$ otherwise, is a restrained 2-rainbow dominating function of $T$ of weight less than $\omega(f)$ and so $b_{r r 2}(T) \leq|B| \leq(n-3) / 2$. All in all, we have $b_{r r 2}(T) \leq(n-3) / 2$ as desired

To prove sharpness, let $T$ be a wounded spider obtained from the star $K_{1, t},(t \geq 3)$ by subdividing $t-2$ edges. It is easy to see that $n(T)=2 t-1, \gamma_{r r 2}(T)=2 t-2$ and $b_{r r 2}(T)=t-2=$ $(n-3) / 2$.

Theorem 2.3. Let $x y z$ be a path of length 2 in the $\operatorname{graph} G$ with $\delta(G) \geq 2$ and $\operatorname{deg}(y) \geq 3$. Then

$$
b_{r r 2}(G) \leq \operatorname{deg}(x)+\operatorname{deg}(y)+\operatorname{deg}(z)-4-\pi(x, z)
$$

where $\pi(x, z)=1$ if $x z \in E(G)$ and $\pi(x, z)=0$ otherwise.

Proof. Let $X \subseteq E(G)$ be the set consisting of all the edges incident with $x, y$ or $z$ with exception of the edges $x y, y z$. Clearly, $|X|=\operatorname{deg}(x)+\operatorname{deg}(y)+\operatorname{deg}(z)-4$ when $x z \notin E(G)$ and $|X|=\operatorname{deg}(x)+$ $\operatorname{deg}(y)+\operatorname{deg}(z)-5$ when $x z \in E(G)$. Let $G_{1}=G-X$ be the graph obtained from $G$ by removing the edges of $X$. In $G_{1}$, the path $x y z$ is a component. We show that $\gamma_{r r 2}\left(G_{1}\right)>\gamma_{r r 2}(G)$ implying that $b_{r r 2}(G) \leq|X|$. Let $f$ be a $\gamma_{r r 2}\left(G_{1}\right)$-function. Since the path $x y z$ is a component of $G_{1}$, we have $|f(x)|+|f(y)|+|f(z)|=3$. If $x z \in E(G)$, then define $g: V(G) \rightarrow \mathscr{P}(\{1,2\})$ by $g(y)=\{1,2\}$, $g(x)=g(z)=\varnothing$ and $g(w)=f(w)$ for $w \in V(G)-\{x, y, z\}$. It is easy to see that $g$ is a restrained 2-rainbow dominating function of $G$ of weight less than $\omega(f)$ as desired.

Now let $x z \notin E(G)$. If there exists a vertex $u \in N(y)-\{x, z\}$ with $f(u)=\varnothing$, then the function $g: V(G) \rightarrow \mathscr{P}(\{1,2\})$ defined by $g(x)=\{1\}, g(z)=\{2\}, g(y)=\varnothing$ and $g(w)=f(w)$ for $w \in V(G)-\{x, y, z\}$, is a restrained 2-rainbow dominating function of $G$ of weight less than $\omega(f)$ as desired. If there are two vertices $u \in N(x)-\{y\}$ and $v \in N(z)-\{y\}$ with $f(u)=f(v)=$ $\varnothing$, then define $g: V(G) \rightarrow \mathscr{P}(\{1,2\})$ by $g(y)=\{1,2\}, g(x)=g(z)=\varnothing$ and $g(w)=f(w)$ for $w \in V(G)-\{x, y, z\}$. Clearly, $g$ is a restrained 2-rainbow dominating function of $G$ of weight less than $\omega(f)$ as desired. Since $\delta(G) \geq 2$, we thus can assume that $f\left(x_{1}\right) \neq \varnothing$ for a vertex $x_{1} \in N(x)-\{y\}$ or $f\left(z_{1}\right) \neq \varnothing$ for a vertex $z_{1} \in N(z)-\{y\}$, say $f\left(x_{1}\right) \neq \varnothing$ for a vertex $x_{1} \in N(x)-\{y\}$. In addition, we may assume that $f(u) \neq \varnothing$ for each vertex $u \in N(y)-\{x, z\}$. Suppose that $u_{1} \in N(y)-\{x, z\}$ (possibly $u_{1}=x_{1}$ ). Assume, without loss of generality, that $1 \in f\left(u_{1}\right)$. Define $g: V(G) \rightarrow \mathscr{P}(\{1,2\})$ by $g(x)=g(y)=\varnothing, g(z)=\{2\}, g\left(x_{1}\right)=\{1,2\}$, and $g(w)=f(w)$ for $w \in V(G)-\left\{x, y, z, x_{1}\right\}$. It is easy to see that $g$ is a restrained 2-rainbow dominating function of $G$ with weight less than $\omega(f)$ as desired. This completes the proof. 
Corollary 2.4. Let $G$ be a connected graph of order $n \geq 6$ with $\delta(G) \geq 2$. Then

$$
b_{r r 2}(G) \leq \delta(G)+2 \Delta(G)-4
$$

The bound is sharp for cycles $C_{3 k+1}$ and $C_{3 k+2}$ where $k \geq 2$.

Proof. If $\Delta(G)=2$, then the result is immediate by Corollary 1.2. Let $\Delta(G) \geq 3$. Assume that $x$ is a vertex of minimum degree $\delta(G)$ such that $x$ is adjacent to a vertex $y$ of degree greater than $\delta(G)$. Since $\operatorname{deg}(y) \geq 3$, there is a path $x y z$ in $G$ satisfying the condition of Theorem 2.3 and the result follows by Theorem 2.3.

Proposition 2.5. Let $x y z$ be a path of length 2 in the graph $G$ such that $\left(N_{G}(x) \cap N_{G}(y)\right) \backslash\{z\} \neq \varnothing$. Then

$$
b_{r r 2}(G) \leq \operatorname{deg}(x)+\operatorname{deg}(y)+\operatorname{deg}(z)-\left|N_{G}(x) \cap N_{G}(y)\right|-\left|N_{G}(x) \cap N_{G}(z)\right|-2-\pi(x, z)
$$

where $\pi(x, z)=1$ if $x z \in E(G)$ and $\pi(x, z)=0$ otherwise.

Proof. Let $X \subseteq E(G)$ be the set consisting of all the edges incident with $x, y$ or $z$ with exception of all edges between $y$ and $N_{G}(x)-\{z\}$ and all edges between $z$ and $N_{G}(x)-\{y\}$. Clearly, $|X|=$ $\operatorname{deg}(x)+\operatorname{deg}(y)+\operatorname{deg}(z)-\left|N_{G}(x) \cap N_{G}(y)\right|-\left|N_{G}(x) \cap N_{G}(z)\right|-3$ if $x z \in E(G)$ and $|X|=\operatorname{deg}(x)+$ $\operatorname{deg}(y)+\operatorname{deg}(z)-\left|N_{G}(x) \cap N_{G}(y)\right|-\left|N_{G}(x) \cap N_{G}(z)\right|-2$ when $x z \notin E(G)$. Let $G_{1}=G-X$ be the graph obtained from $G$ by removing the edges of $X$. In $G_{1}$, the vertex $x$ is isolated and the neighbors of $y$ or $z$ lie in $N_{G}(x)$. We show that $\gamma_{r r 2}\left(G_{1}\right)>\gamma_{r r 2}(G)$ that implies $b_{r r 2}(G) \leq|X|$ as desired. Let $f$ be a $\gamma_{r r 2}\left(G_{1}\right)$-function. Since $x$ is isolated in $G_{1}$, we may assume that $f(x)=\{1\}$. If $f(y)=\varnothing$, then the function $g: V(G) \rightarrow \mathscr{P}(\{1,2\})$ defined by $g(x)=\varnothing$ and $g(w)=f(w)$ for $w \in V(G)-\{x\}$, is a restrained 2-rainbow dominating function of $G$ of weight less than $\omega(f)$ as desired. Assume that $|f(y)| \geq 1$. If $f(y)=\{1,2\}$, then the function $g: V(G) \rightarrow \mathscr{P}(\{1,2\})$ defined by $g(y)=\{2\}$ and $g(w)=f(w)$ for $w \in V(G)-\{y\}$, is a restrained 2-rainbow dominating function of $G$ with weight less than $\omega(f)$ as desired.

Let $|f(y)|=1$. Assume, without loss of generality, that $f(y)=\{1\}$. If $f(z)=\varnothing$, then the function $g: V(G) \rightarrow \mathscr{P}(\{1,2\})$ given by $g(x)=\varnothing$ and $g(w)=f(w)$ for $w \in V(G)-\{x\}$ is a restrained 2-rainbow dominating function of $G$ with weight less than $\omega(f)$ as desired. Suppose $|f(z)| \geq 1$. If there exists a vertex $w_{1} \in\left(N_{G}(x) \cap N_{G}(y)\right)$ with $f\left(w_{1}\right)=\varnothing$, then the function $g$ defined by $g(x)=\{1\}, g(z)=\{2\}, g(y)=\varnothing$ and $g(w)=f(w)$ for $w \in V(G)-\{x, y, z\}$ is a restrained 2-rainbow dominating function of $G$ with weight less than $\omega(f)$ as desired. Hence, we assume $f(w) \neq \varnothing$ for each $w \in\left(N_{G}(x) \cap N_{G}(y)\right)-\{z\}$. Assume that $w \in\left(N_{G}(x) \cap N_{G}(y)\right)-\{z\}$. Define the function $g$ by $g(w)=\{1,2\}, g(x)=g(y)=\varnothing$ and $g(a)=f(a)$ for $a \in V(G)-\{w, x, y\}$. Clearly, $g$ is a restrained 2-rainbow dominating function of $G$ with weight less than $\omega(f)$ as desired. 
Thus $\gamma_{r r 2}\left(G_{1}\right)>\gamma_{r r 2}(G)$ implying that $b_{r r 2}(G) \leq|X|$ and the proof is complete.

\section{Complete graphs and complete bipartite graphs}

In this section, we determine the restrained 2-rainbow bondage number of complete graphs and complete bipartite graphs.

Observation 3.1. For every graph $G$ with $\gamma_{r r 2}(G)=\gamma_{r 2}(G)<n, b_{r 2}(G) \geq b_{r r}(G)$.

Proof. For any $b_{r 2}(G)$-set $B$, we have $\gamma_{r r 2}(G-B) \geq \gamma_{r 2}(G-B)>\gamma_{r 2}(G)=\gamma_{r r 2}(G)$ that implies $b_{r 2}(G) \geq b_{r r 2}(G)$.

Proposition 3.2. For $n \geq 9$,

$$
b_{r r 2}\left(K_{n}\right)=\left\lceil\frac{2 n}{3}\right\rceil .
$$

Proof. By Observation 3.1 and Theorem E, we have $b_{r r 2}\left(K_{n}\right) \leq\left\lceil\frac{2 n}{3}\right\rceil$. Now we show that $b_{r r 2}\left(K_{n}\right) \geq\left\lceil\frac{2 n}{3}\right\rceil$. Let $V\left(K_{n}\right)=\left\{x_{1}, x_{2}, \ldots, x_{n}\right\}$ be the vertex set of $K_{n}$ and let $B$ be a $b_{r r 2}\left(K_{n}\right)$ set. Assume, to the contrary, that $|B|<\left\lceil\frac{2 n}{3}\right\rceil$. It follows from Theorem E that $\gamma_{r 2}\left(K_{n}-B\right)=2$. By Theorem F, $\Delta\left(K_{n}-B\right)=n-1$ or there are two vertices, say $x_{1}, x_{2}$, such that $\left\{x_{3}, \ldots, x_{n}\right\} \subseteq$ $N\left(x_{1}\right) \cap N\left(x_{2}\right)$. First let $\Delta\left(K_{n}-B\right)=n-1$ and $\operatorname{deg}_{K_{n}-B}\left(x_{1}\right)=n-1$. If $\delta\left(K_{n}-B\right) \geq 2$, then clearly $\gamma_{r r 2}\left(K_{n}-B\right)=2$ which is a contradiction. Hence, $\operatorname{deg}_{K_{n}-B}\left(x_{i}\right)=1$ for some $2 \leq i \leq n$ that implies $|B| \geq n-2 \geq\left\lceil\frac{2 n}{3}\right\rceil$, a contradiction. Now let $\Delta\left(K_{n}-B\right) \leq n-2$. Then $\left\{x_{3}, \ldots, x_{n}\right\} \subseteq$ $N\left(x_{1}\right) \cap N\left(x_{2}\right)$. If $\delta\left(K_{n}-B\right) \geq 3$, then clearly $\gamma_{r r 2}\left(K_{n}-B\right)=2$ which is a contradiction. Assume that $\operatorname{deg}_{K_{n}-B}\left(x_{i}\right)=2$ for some $3 \leq i \leq n$, say $i=3$. It follows that $\left\{x_{3} x_{4}, \ldots, x_{3} x_{n}\right\} \subseteq B$. Since $\Delta\left(K_{n}-B\right) \leq n-2$, we deduce that $x_{1} x_{2} \in B$ and so $|B| \geq n-2 \geq\left\lceil\frac{2 n}{3}\right\rceil$, a contradiction. Thus $b_{r r 2}\left(K_{n}\right)=\left\lceil\frac{2 n}{3}\right\rceil$ and the proof is complete.

Proposition 3.3. For $3 \leq n \leq 8$,

$$
b_{r r 2}\left(K_{n}\right)=n-2 \text {. }
$$

Proof. If $n=3$, then we have $b_{r r 2}\left(K_{3}\right)=1$. Let $n \geq 4$ and $V\left(K_{n}\right)=\left\{x_{1}, x_{2}, \ldots, x_{n}\right\}$ be the vertex set of $K_{n}$. It follows from Theorem D that $\gamma_{r r 2}\left(K_{n}-\left\{x_{1} x_{2}, \ldots, x_{1} x_{n-1}\right\}\right) \geq 3$. Also the function $f$ defined by $f\left(x_{1}\right)=\{1\}, f\left(x_{2}\right)=\{1,2\}$ and $f(x)=\varnothing$ otherwise, is an R2RDF of $K_{n}-B$ of weight 3 that implies $\gamma_{r r 2}\left(K_{n}-\left\{x_{1} x_{2}, \ldots, x_{1} x_{n-1}\right\}\right)=3>\gamma_{r r 2}\left(K_{n}\right)$. Hence $b_{r r 2}\left(K_{n}\right) \leq n-2$. If $n=4$, then it follows from Theorem D that $\gamma_{r r 2}\left(K_{4}-e\right)=2$ for each $e \in E\left(K_{4}\right)$ and so $b_{r r 2}\left(K_{4}\right)=2$. If $n=5$, then clearly for every two edges $e_{1}, e_{2} \in E\left(K_{5}\right)$, we have $\Delta\left(K_{5}-\left\{e_{1}, e_{2}\right\}\right)=4$ and $\delta\left(K_{5}-\left\{e_{1}, e_{2}\right\}\right) \geq$ 2. Hence, $\gamma_{r r 2}\left(K_{5}-\left\{e_{1}, e_{2}\right\}\right)=2$ by Theorem D that implies $b_{r r 2}\left(K_{5}\right)=3$. Let $6 \leq n \leq 8$. Since $n-2=\left\lceil\frac{2 n}{3}\right\rceil$, using an argument similar to that described in the proof of Proposition 3.2 leads to $b_{r r 2}\left(K_{n}\right)=n-2$. 
Proposition 3.4. For integers $2 \leq p \leq q$ and $q \neq 2$,

$$
b_{r r 2}\left(K_{p, q}\right)= \begin{cases}2 & \text { if } p=2 \\ 3 & \text { if } p=3 \\ p-1 & \text { otherwise. }\end{cases}
$$

Proof. Let $X=\left\{x_{1}, x_{2}, \ldots, x_{p}\right\}$ and $Y=\left\{y_{1}, y_{2}, \ldots, y_{q}\right\}$ be the partite sets of $K_{p, q}$, and let $B$ be a $b_{r r 2}\left(K_{p, q}\right)$-set. We note that $\gamma_{r r 2}\left(K_{p, q}\right)=4$ for $q \geq p \geq 2$.

First let $p=2$. For any edge $x_{i} y_{j} \in E\left(K_{2, q}\right)$, say $i=1, j=1$, the function $f: V\left(K_{2, q}\right) \rightarrow$ $\mathscr{P}(\{1,2\})$ defined by $f\left(y_{1}\right)=\{1\}, f\left(y_{2}\right)=\{2\}, f\left(x_{1}\right)=\{1,2\}$ and $f(u)=\varnothing$ otherwise, is a $\gamma_{r r 2}\left(K_{2, q}-x_{1} y_{1}\right)$-function and so $\gamma_{r r}\left(K_{p, q}-x_{1} y_{1}\right)=4$. It follows that $b_{r r 2}\left(K_{2, q}\right) \geq 2$. On the other hand, it is clear that $\gamma_{r r 2}\left(K_{2, q}-\left\{x_{1} y_{1}, x_{2} y_{1}\right\}\right)=5$ that implies $b_{r r 2}\left(K_{2, q}\right)=2$.

Now let $p=3$. Let $e_{1}, e_{2}$ be two arbitrary distinct edges of $K_{3, q}$. If $e_{1}$ and $e_{2}$ have a common endpoint, then we may assume, without loss of generality, that $e_{1}=x_{1} y_{1}, e_{2}=x_{2} y_{1}$ or $e_{1}=$ $x_{1} y_{1}, e_{2}=x_{1} y_{2}$, and if $e_{1}$ and $e_{2}$ have no common endpoint, then we may assume that $e_{1}=$ $x_{1} y_{1}$ and $e_{2}=x_{2} y_{2}$. If $e_{1}=x_{1} y_{1}, e_{2}=x_{2} y_{1}$ or $e_{1}=x_{1} y_{1}, e_{2}=x_{1} y_{2}$ and $q=3$ or $e_{1}=x_{1} y_{1}, e_{2}=$ $x_{2} y_{2}$ then define $f: V\left(K_{3, q}\right) \rightarrow \mathscr{P}(\{1,2\})$ by $f\left(x_{1}\right)=f\left(y_{1}\right)=\{1\}, f\left(x_{2}\right)=f\left(y_{2}\right)=\{2\}$ and $f(u)=\varnothing$ otherwise, and if $e_{1}=x_{1} y_{1}, e_{2}=x_{1} y_{2}$ and $q \geq 4$ then define $f: V\left(K_{3, q}\right) \rightarrow \mathscr{P}(\{1,2\})$ by $f\left(x_{3}\right)=$ $f\left(y_{q}\right)=\{1,2\}$ and $f(u)=\varnothing$ otherwise. It is easy to see that $f$ is a $\gamma_{r r 2}\left(K_{3, q}-\left\{e_{1}, e_{2}\right\}\right)$-function of weight 4 and hence $b_{r r 2}\left(K_{3, q}\right) \geq 3$. It is easy to see that $\gamma_{r r 2}\left(K_{3, q}-\left\{x_{1} y_{1}, x_{2} y_{1}, x_{3} y_{1}\right\}\right)=5$ that implies $b_{r r 2}\left(K_{3, q}\right)=3$.

Finally let $p \geq 4$. Considering $B=\left\{x_{2} y_{1}, x_{3} y_{1}, \ldots, x_{p} y_{1}\right\}$, one can see that $\operatorname{deg}_{K_{p, q}-B}\left(y_{1}\right)=1$ and $\gamma_{r r 2}\left(K_{p, q}-B\right)=5$. It follows that $b_{r r 2}\left(K_{p, q}\right) \leq p-1$. Now we show that $b_{r r 2}\left(K_{p, q}\right) \geq$ $p-1$. Let $F$ be an arbitrary set of edges with $|F| \leq p-2$. It is clear that $\delta\left(K_{p, q}-F\right) \geq 2$ and $\operatorname{deg}_{K_{p, q}-F}\left(x_{i_{1}}\right)=\operatorname{deg}_{K_{p, q}-F}\left(x_{i_{2}}\right)=q, \operatorname{deg}_{K_{p, q}-F}\left(y_{j_{1}}\right)=\operatorname{deg}_{K_{p, q}-F}\left(y_{j_{2}}\right)=p$ for some $1 \leq i_{1}, i_{2} \leq$ $p$ and $1 \leq j_{1}, j_{2} \leq q$. Define $f: V\left(K_{p, q}\right) \rightarrow \mathscr{P}(\{1,2\})$ by $f\left(x_{i_{1}}\right)=f\left(y_{j_{1}}\right)=\{1,2\}$ and $f(u)=\varnothing$ otherwise. It is easy to see that $f$ is a $\gamma_{r r 2}\left(K_{p, q}-B\right)$-function of weight 4 and hence $b_{r r 2}\left(K_{p, q}\right) \geq$ $p-1$. Thus $b_{r r 2}\left(K_{p, q}\right)=p-1$ when $p \geq 4$ and the proof is complete.

\section{References}

[1] H. A. Ahangar, J. Amjadi, V. Samodivkin, S. M. Sheikholeslami and L. Volkmann, On the rainbow restrained domination number, Ars Combin., 125 (2016), 209-224.

[2] J. Amjadi, L. Asgharshrgi, N. Dehgardi, M. Furuya, S. M. Sheikholeslami and L. Volkmann, The k-rainbow reinforcement numbers in graphs, Discrete Appl. Math., 217 (2017), 394-404.

[3] J. Amjadi, N. Dehgardi, M. Furuya and S. M. Sheikholeslami, A sufficient condition for large rainbow domination number, International Journal of Computer Mathematics: Computer Systems Theory, 2 (2017), 53-65.

[4] J. Amjadi and A. Parnian, On the 2-rainbow bondage number of planar graphs, Ars Combin., 126 (2016), 395-405. 
[5] J. Amjadi, S. M. Sheikholeslami and L. Volkmann, Rainbow restrained domination numbers in graphs, Ars Combin., 124 (2016), 3-19.

[6] L. Asgharsharghi, S. M. Sheikholeslami and L. Volkmann, A note on the 2-rainbow bondage numbers in graphs, Asian-European J. Math., 9 (2016), 1650013 (7 pages)

[7] B. Brešar, M.A. Henning, and D.F. Rall, Rainbow domination in graphs, Taiwanese J. Math., 12 (2008), 213225.

[8] B. Brešar, and T.K. Šumenjak, On the 2-rainbow domination in graphs, Discrete Appl. Math., 155 (2007), 2394-2400.

[9] G. J. Chang, J. Wu and X. Zhu, Rainbow domination on trees, Discrete Appl. Math., 158 (2010), 8-12.

[10] T. Chunling, L. Xiaohui, Y. Yuansheng and L. Meiqin, 2-rainbow domination of generalized Petersen graphs $P(n, 2)$, Discrete Appl. Math., 157 (2009), 1932-1937.

[11] N. Dehgardi, S. M. Sheikholeslami and L. Volkmann, The k-rainbow bondage number of a graph, Discrete Appl. Math., 174 (2014), 133-139.

[12] G. S. Domke, J. H. Hattingh, S. T. Hedetniemi and L. R. Markus, Restrained domination in trees, Discrete Math., 211 (2000), 1-9.

[13] G. S. Domke, J. H. Hattingh, M. A. Henning and L. R. Markus, Restrained domination in graphs with minimum degree two, J. Combin. Math. Combin. Comput., 35 (2000), 239-254.

[14] G. S. Domke, J. H. Hattingh, S. T. Hedetniemi, R. C. Laskar, and L. R. Markus, Restrained domination in graphs, Discrete Math., 203 (1999), 61-69.

[15] J.H. Hattingh and A.R. Plummer, Restrained bondage in graphs, Discrete Math., 308 (2008), 5446-5453.

[16] B. Hartnell and D. F. Rall, On dominating the Cartesian product of a graph and $K_{2}$, Discuss. Math. Graph Theory, 24 (2004), 389-402.

[17] T. W. Haynes, S. T. Hedetniemi and P. J. Slater, Fundamentals of Domination in Graphs, Marcel Dekker, Inc. New York, 1998.

[18] R. Kala and T. R. Nirmala Vasantha, Restrained bondage number of a graph, J. Discrete Math. Sci. Cryptogr., 12 (2009), 373-380.

[19] D. Meierling, S. M. Sheikholeslami and L. Volkmann, Nordhaus-Gaddum bounds on the k-rainbow domatic number of a graph, Appl. Math. Lett., 24 (2011), 1758-1761.

[20] Z. Shao, M. Liang, C. Yin, X. Xu, P. Pavlič and J. Žerovnik, On rainbow domination numbers of graphs, Information Sciences, 254 (2014), 225-234.

[21] S. M. Sheikholeslami and L. Volkmann, The k-rainbow domatic number of a graph, Discuss. Math. Graph Theory, 32 (2012), 129-140.

[22] D. B. West, Introduction to Graph Theory, Prentice-Hall, Inc, 2000.

[23] Y. Wu and N. Jafari Rad, Bounds on the 2-rainbow domination number of graphs, Graphs Combin., 29 (2013), 1125-1133.

[24] G. Xu, 2-rainbow domination of generalized Petersen graphs $P(n, 3)$, Discrete Appl. Math., 157 (2009), $2570-$ 2573.

Department of Mathematics, Azarbaijan Shahid Madani University, Tabriz, I.R. Iran.

E-mail: j-amjadi@azaruniv.edu

Department of Mathematics and Computer Science, Sirjan University of Technology, Sirjan, I.R. Iran.

E-mail: ndehgardi@gmail.com

Department of Mathematics, Azarbaijan Shahid Madani University, Tabriz, I.R. Iran.

E-mail: khoeilar@azaruniv.edu

Department of Mathematics, Azarbaijan Shahid Madani University, Tabriz, I.R. Iran.

E-mail: s.m.sheikholeslami@azaruniv.edu

Lehrstuhl II für Mathematik, RWTH Aachen University, 52056 Aachen, Germany.

E-mail: volkm@math2.rwth-aachen.de 